\title{
Unilateral Sensorineural Hearing Loss in the Southern Region of Saudi Arabia
}

\author{
Tawfiq Khurayzi ${ }^{1 *}$, Abdullah AlSkaini ${ }^{2}$ and Ibrahim Sumaily ${ }^{1}$ \\ ${ }^{1}$ Otolaryngology Department, King Fahd Central Hospital, Jizan, KSA \\ ${ }^{2}$ Otolaryngology Department, Asir Central Hospital, Abha, KSA
}

Submission: May 08, 2018; Published: May 17, 2018

*Corresponding author: Tawfiq Khurayzi, Department of otolaryngology, King Fahd Central Hospital Jizan, KSA, Email: tawfiqaakk@hotmail.com

\section{Abstract}

Background: Unilateral sensorineural hearing loss (USNHL) is important to be identified and diagnosed early. It is common and can be a symptom of a wide range of diseases. In our region, southern region of Saudi Arabia, the USNHL has not been studied before. In this study we report the incidence and common presentation and causes of this entity.

Method and Aim: A retrospective chart review of 209 patient with USNHL whom underwent full investigations in adult age group. We aim to find the risk factors, causes, common presentation, diagnostic work up and prognosis of USNHL in the southern region of Saudi Arabia and comparing the result with a literature review.

Result: Out of 209 patients, 183 met our criteria. Most of our sample had chronic mode hearing loss (74.8\%). Tinnitus, vertigo, aural fullness and ear pain were the most common associated symptoms respectively. On audiological assessment: PTA at time of investigation, half of them at high frequency (54\%) and severe hearing loss (31.1\%). Speech discrimination was very poor in all patients with retrocochlear pathology. ABR done in all cases with suspected to have retrocochlear lesion with positive finding affected on wave V. All patients underwent magnetic resonance imaging and is was normal in most of them (75.4\%). While in 3.8\% there was retrocochlear lesion, (5\%) there was cochlear lesion, and in (13.6\%) there was central nervous system pathology. Common diagnoses found to be idiopathic SSNHL (14.7\%), NIHL (13.3\%), Meniere's disease $(14.7 \%)$, presbycusis $(14.5 \%)$, central nerves system $(11.4 \%)$, head trauma $(8.2 \%)$, ototoxic medication $(6.5 \%)$, infection $(3.8 \%)$, cerebellopontine angle (2.7\%), metabolic/autoimmune (1\%) and congenital $(0.5 \%)$.

Conclusion: All patients with USNHL need full audiological screening and early diagnosis. Regarding our study, we recommend MRI as part of their diagnostic workup. The choice of diagnostic laboratory tests should be directed by patient risk factors and exposures.

Keywords: Unilateral sensorineural hearing loss; Single side hearing loss; Sensorineural hearing loss

\section{Introduction}

Hearing disorders it can divide to sensorineural hearing loss (SNHL) and conductive hearing loss (CHL). SNHL is a common disorder that results from damage to the inner ear in over $95 \%$ of all cases. Therefore, retrocochlear hearing disorders are rare and cannot be differentiated from sensory losses by clinical symptoms alone. Unilateral hearing loss affect the quality of life in the affected patients. Conductive deafness has a readily determined etiology in most cases. In contrast, USNHL (USNHL) requires more refined and extensive investigation [1]. There are very few studies discussing the incidence of USNHL. SNHL, which accounts for $90 \%$ of the cases of Americans people those presenting with hearing loss and it can be caused by damage to the cochlea or the vestibule-cochlear nerve. The vast majority of patients with SNHL have bilateral hearing loss and on the other hand, approximately 60,000 new cases of USNHL occur annually in the United States 2. According to the World Health Organization (WHO), Hearing defects are the most frequent sensory deficit in human population, affecting more than 250 million people in the world with an average of 7 out of 100 of the population in Saudi Arabia and still need more and more searching about prevalence of USNHL. It is important to identify and diagnose it early. It occurs when the hearing in one ear is within normal hearing capacity and the other ear has a hearing loss. The normal audiometric function is defined as hearing thresholds that are not poorer than 20-25 dB hearing level (HL) for pure-tone audiometry (PTA).

The diseased ear can be range above that, above $25 \mathrm{~dB}$, from mild to profound level hearing loss. Because a wide variety of pathologic processes may be responsible for the USNHL, numerous diagnostic tests are usually used during initial 


\section{Global Journal of Otolaryngology}

evaluation, including pure-tone audiometry, acoustic reflex testing, imaging, serologic testing, and auditory brainstem response testing 1,8. USNHL can be a symptom of a wide range of diseases. Also, can be defined by site of disease, severity, audiometric configuration, and method of onset. The most frequent mechanism cause of USNHL or single-sided deafness (SSD) are sudden deafness; Meniere's disease; cerebellopontine angle tumors / vestibular schwannoma (acoustic neuroma); neoplasms; demyelinating pathologies such as multiple sclerosis, vertebrobasilar arterial occlusion, stroke; acoustic trauma; head injury; perilymphatic fistula; ototoxic drugs; labyrinthitis; autoimmune disease as Cogan disease, Wegener's granulomatosis, lupus, Takayasu arteritis, systemic sclerosis, and other rheumatological disorders and idiopathic SNHL 3,4,14.

Aim: Our aim is to find the risk factors and causes of USNHL, presenting symptoms, diagnostic work up and prognosis in southern region of Saudi Arabia and comparing the result with literatures.

Method: A retrospective chart review for last 10 years (2006-2016 G) in Asir Central hospital, a tertiary hospital in the southern region of Saudi Arabia. Medical record collected and reviewed for 209 patients with USNHL whom underwent full investigations at time of presentation and with closed audiological follow up in clinic for 1 years post first presentation of hearing loss in different adult age groups. We are excluded pediatric age group less than 12 years, patients with contralateral hearing loss, those with ipsilateral mixed and conductive hearing loss and who has history of ear surgery. Data analysis done with SPSS v.22.

Result: 183 patients met our criteria, 108 males (59\%) and 75 females (41\%). Onset and Duration; Most of them had gradual onset of hearing loss, (74.8\%). while (25.2\%) reported sudden onset. At the time of presentation, in 100 cases (54.6\%) the hearing loss was prolonged for more than six months duration, $45(24.6 \%)$ cases from 30 days to 6 months, and 38 cases $(20.7 \%)$ presented within one month of the inset.

History of Risk factors; 12 cases (6.5\%) of the 183 total patients had mechanical head trauma, 16 patients $(8.7 \%)$ received ototoxic medications, and 16 patients $(8.7 \%)$ with positive criteria for exposed to risky noise. Only (35.5\%) had chronic medical illness. The association symptoms were tinnitus in 135 cases (73.7\%), vertigo in 121 cases (66.1\%), aural fullness in 36 cases (19.6\%), pain in 28 cases $(15.3 \%)$. Audiological assessment; pure tone audiometry (PTA) at time of investigation and during audiological follow up, most of them, 99 cases (54\%) at high frequency $4 \mathrm{kHz}$ and above, 20 cases $(11 \%)$ at low frequency less than $1 \mathrm{kHz}, 18$ cases $(9.8 \%)$ at middle frequency $1 \mathrm{kHz}-2 \mathrm{kHz}$ and 46 cases (25\%) at all frequency. On the other hand, 57 cases $(31.1 \%)$ in severe level, 42 cases $(23 \%)$ in moderate level, 33 cases $(18 \%)$ in moderatesevere level, 33 cases $(18 \%)$ at mild level, 13 cases $(7.1 \%)$ at profound and 5 cases (2.8\%) as dead ear. See Figure 1.

\begin{tabular}{|c|c|c|c|c|c|c|c|}
\hline \multicolumn{2}{c}{ Mild } & Moderate & $\begin{array}{c}\text { Moderately- } \\
\text { Sever }\end{array}$ & Sever & Profound & Dead ear & Total \\
\hline Male & $15(8.1 \%)$ & $24(13.1 \%)$ & $14(7.6 \%)$ & $45(24.5 \%)$ & $8(4.37 \%)$ & $2(1 \%)$ & $108(59 \%)$ \\
\hline Female & $18(9.8 \%)$ & $18(9.8 \%)$ & $19(10.4 \%)$ & $12(6.5 \%)$ & $5(2.7 \%)$ & $3(1.6 \%)$ & $75(41 \%)$ \\
\hline Total & $33(18 \%)$ & $42(22.9 \%)$ & $33(18 \%)$ & $57(31.1 \%)$ & $13(7.1 \%)$ & $5(2.6 \%)$ & 183 \\
\hline
\end{tabular}

Noise induced hearing loss notch at $4000 \mathrm{~Hz}$ found in 20 cases $(11 \%)$ only. Speech discrimination which is ability to understand speech with a list of phonetically balanced words was done only in 141 patients as the following: normal in (10\% of 141), slight difficulty in (15.5\% of 141$)$, moderate difficulty (28.4\% of 141$)$, poor in (25.7\% of 141$)$, and very poor in $(20.4 \%$ of 141). In addition, Speech discrimination was very poor result was in all patients with retrocochlear pathology. Auditory brainstem response (ABR) done in all cases suspected to have retrocochlear lesion and there were positive findings: 11 cases (6\%) retrocochlear lesion and 32 cases $(17.5 \%)$ was cochlear SNHL. MRI done for all of the patients and it was normal in 138 cases $(75.4 \%)$, while in 9 cases $(5 \%)$ it shows cochlear lesion, 7 cases (3.8\%) it shows retrocochlear lesion including cerebellopontine angle lesion 5 cases (2.7\%). Also, 4 cases $(2.2 \%)$ with middle ear pathology and $(13.6 \%)$ have central nerves system pathology Figure 2.

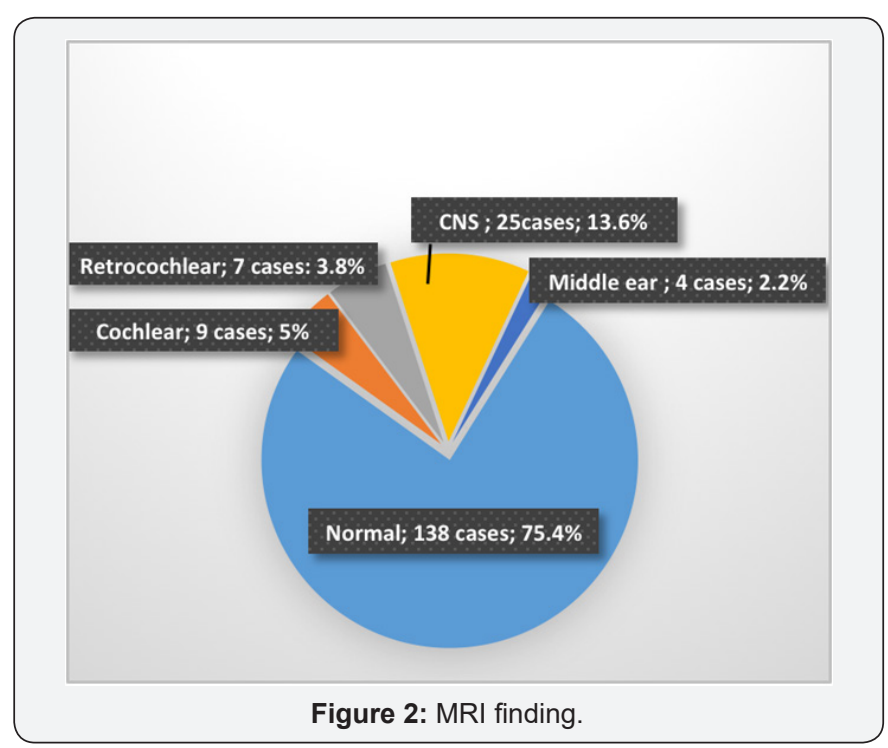


We are categorized the Diagnosis as following: noise induced hearing loss in 25 cases (13.6\%), Meniere's disease in 26 cases (14.7\%), presbycusis in 27 cases (14.5\%), central nerves system pathology in 21 cases (11.4\%), cochlear otosclerosis 15 cases (8.2\%), idiopathic sudden sensorineural hearing loss (SSNHL)
27 cases $(14.7 \%)$, head trauma 15 cases (8.2\%), ototoxic medication SNHL 12 cases $(6.5 \%)$, infection 7 cases $(3.8 \%)$, cerebellopontine angle lesion 5 cases (2.7\%), metabolic/ autoimmune 2 cases $(1 \%)$ and congenital 1 cases $(0.5 \%)$ Figure 3.

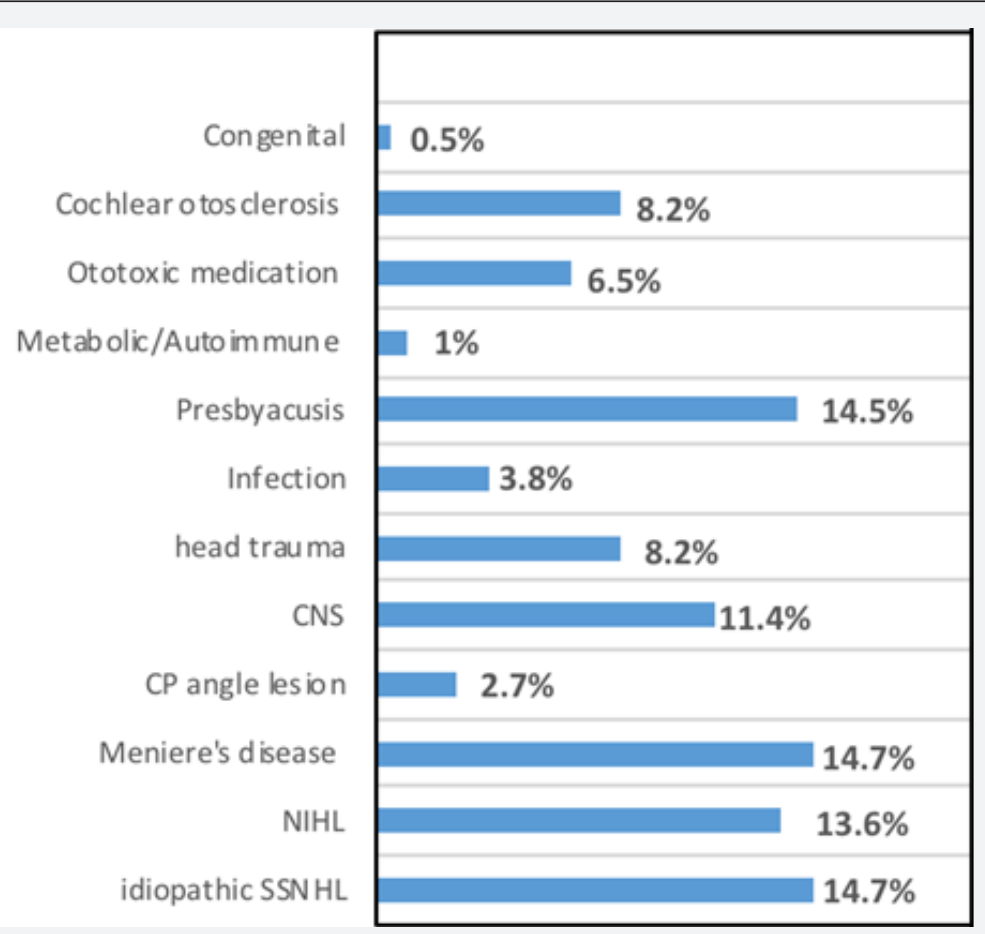

Figure 3: Common causes of USNHL.

\section{Discussion}

Hearing loss is an extremely common disorder. Most of patients with SNHL have bilateral hearing loss and on the other hand, approximately 60,000 new cases of USNHL occur annually in the United States 2. According to the World Health Organization (WHO), Hearing defects are the most frequent sensory deficit in human populations, affecting more than 250 million people in the world with an average of 7 out of 100 of the population in Saudi Arabia. Few studies on USNHL are available in the literature and database. We are concern in our study on adult patients group. Also, in both sex, in our result the USNHL is more common in male than female, which is running in same configuration with the WHO Deafness and HL Fact Sheet No. 300 (2015). In our study, most of the patients presented with severe level hearing loss (31.1\%) and (23\%) in moderate level severity. Moreover, some patients can present with moderatesevere level (18\%), mild level (18\%) and (7.1\%) at profound as in our study. When we comparing with other studies, common unilateral hearing loss ranged from mild (34.2\%) to profound (31.96\%) [16].

Unilateral hearing losses in children have traditionally been underappreciated, but health care practitioners are now beginning to understand their effect on development and the underlying pathophysiologic mechanisms. Average age of diagnosis unilateral SNHL is 8.7 years. 3/1000 Unilateral SNHL greater than $50 \mathrm{~dB}$, and Genetic cause in $50 \%$ of individuals 38 . Universal infant screening has led to earlier diagnosis. On the other hand, congenital hearing loss may be delayed. In criteria of our study we are exclude all children. Moreover, we find 1 case $(0.5 \%)$ diagnosed with unilateral congenital familial hearing loss.

In our study of 183 cases, 137 cases (74.8\%) had chronic mode hearing loss and 46 cases $(25.2 \%)$ in sudden mode of onset. 27 cases $(14.7 \%)$ met the criteria of idiopathic sudden SNHL and we diagnosed him as idiopathic SSNHL. Divyank Bansal et al, he did similar as our study on 155 patients and he find (7.74\%) had sudden onset and (92.26\%) had gradual onset 16. In addition to that, another study published in 2015 on 820 patients with SNHL they find that $5 \%$ presented with sudden HL and $95 \%$ with gradual HL 18. But still there is variation in as in Hughes et al, in the United States; they find that only $1 \%$ of cases are of sudden hearing loss. Sudden SNHL (SSHL) have a strictest definition is a $30 \mathrm{~dB}$ loss over 3 contiguous frequencies occurring within 3 days and it is considered an otological emergency 19.

A number of factors have been identified that contribute to the presentation of unilateral sudden sensorineural hearing loss. Routinely, patients presenting undergo a battery of full investigation to determine a cause. The utility of the 
comprehensive evaluation should be reconsidered. The choice of diagnostic evaluation should be directed by patient risk factors and exposures 6 . SSNHL is most commonly and differential for SSNHL is vast, for the majority of patients an etiologic factor is not identified 7.

In addition to above risk factor, ototoxic medications is considered strong risk, as we find, 16 cases (8.7\%) received ototoxic medications before time of presentation with SNHL and 12 cases $(6.5 \%)$ of those 16 cases developed SNHL within and early after receiving the course of this medication and we diagnose him as SNHL secondary to ototoxicity and all of them present with severe high-frequency SNHL. As we know most ototoxic medications cause bilateral damage and SNHL, but it can cause unilateral hearing loss also. Ototoxic drugs almost cause high-frequency SNHL. The most common drugs with ototoxic effects include aminoglycosides, loop diuretics, salicylates, and chemotherapy agents and the ototoxic effect increased in elderly patients and patients with poor renal function 21 .

25 cases $(13.6 \%)$ diagnosed as Noise-induced hearing loss (NIHL) and positive finding of Noise induced hearing loss notch 20 cases. loud sound is risky to ear. this risk increased by repeated exposure. Usually due to totally or partial damage to the organ of Corti and/or rupture of the cochlear membranes. NIHL it can be a temporary threshold shift followed by a permanent threshold shift after repeated exposure. In contrast, acoustic trauma is caused by a single high-intensity noise exposure that results in a permanent threshold shift without a temporary threshold shift and usually causes a sudden permanent unilateral or asymmetric. An audiogram of a patient with NIHL and acoustic trauma will show a typical noise notch, which is a hearing loss from 3000 to $6000 \mathrm{~Hz}$, with the poorest hearing often centered at $4000 \mathrm{~Hz}$ [21].

Meniere's disease, known as idiopathic endolymphatic hydrops, presents with a combination of hearing loss, aural fullness, tinnitus, and/or vertigo. Meniere's disease accounts for around $5 \%$ of SNHL cases 22 . Comparing to 26 cases $(14.7 \%$ ) in our study and all of them collect the definite or probable criteria of meniere's disease. The diagnosis is usually made by clinical history and audiological hearing tests as we did. However, electrocochleography and cochlear hydrops masking procedure can be used to supplement the clinical history for diagnosis.

Autoimmune/metabolic can also affected on inner ear and cause SNHL bilaterally in most cases but still there are some patients present with unilateral SNHL as in our study: 2 cases (1\%), one known case of systemic lupus erythematosus and other with severe rheumatoid arthritis. Systemic autoimmune disorders associated with SNHL include Cogan syndrome, polyarteritis nodosa, relapsing polychondritis, Wegener's granulomatosis, systemic lupus erythematosus, Takayasu arteritis, systemic sclerosis, and other rheumatological disorders 23.
Cochlear otosclerosis can be classified as a mixed type or a sensorineural type according to the clinical appearance. Cochlear otosclerosis is defined as a focus of otosclerosis located in the otic capsule involving the cochlear endosperm and causing sensorineural hearing loss without any stapes fixation or any conductive component. If the definition of cochlear otosclerosis is accepted as the involvement of cochlear endosperm without associated stapes fixation, then the incidence among ears with pure progressive sensorineural hearing loss is about $1 \% 37$. We collect only $15(8.2 \%)$ cases meting criteria of cochlear otosclerosis.

Infection of inner ear still play important role in unilateral SNHL and almost patient present with vestibular symptoms. Labyrinthitis infection 7 cases (3.8\%), as in our findings. Labyrinthitis can be suppurative or serous. Suppurative Infection can extend into the inner ear either by otogenic routes (acute or chronic otitis media) or meningogenic routes (the IAC or the cochlear/vestibular aqueduct. Infection also may cause bony erosion of the otic capsule. An infection causing meningitis can spread through the cochlear aqueduct or the IAC on the other hand; serous infection is defined as an abnormal process within the labyrinth caused by bacterial toxins, blood contamination of perilymph, or viruses 21 . Common viruses related to unilateral hearing loss include measles, mumps, rubella, varicella zoster and cytomegalovirus. Other infections include syphilis, Rocky Mountain spotted fever, Lyme disease 24,25,26,27.

Presbyacusis is a complex and multifactorial disorder usually characterized by symmetrical progressive loss of hearing over many years and usually affects high frequencies of hearing. Also, can be asymmetry in PTA and speech levels, between the two ears. It is not uncommon to find the sensory loss more pronounced in one ear. We find (14.5\%) 27 patients, all of them above 70 years, have mild to moderate high frequency hearing loss with good speech discrimination in 7 patients and 12 patients mild loss and 8 patients poor discrimination. all of them running with criteria of Presbyacusis after we exclude other causes. Diagnosis is frequently influenced by concurrent aging in other tissues and is frequently established by exclusion. Unilateral tinnitus or a gross asymmetry of response between the two sides frequently suggests pathology other than presbyacusis and warrants further investigation 6 .

Central nerves system pathology can cause unilateral hearing loss. 21 cases $(11.4 \%)$ of patients in our study carried with this diagnosis ether as stroke or demyelinating process according to MRI and neurologist assessment. Multiple sclerosis it common example of demyelination central pathology and can present almost with neurological symptom and between $4 \%-10 \%$ with SNHL 28,29. Hearing loss in multiple sclerosis patients usually present in various patterns, frequently, it can be sudden and unilateral hearing loss $31,32$.

An ABR and MRI play important role to rash to diagnosis 32, $33,34,35$. Stroke (CVA) can affect cochlear and vestibular brain 
stem nuclei through anterior inferior cerebellar artery. As well as the labyrinth through internal auditory artery, which supplies the labyrinth 20 ?

We find that 15 cases (8.2\%) with Mechanical head trauma and all of them had hearing loss developed after trauma. It is considered as risk factor. This trauma to a head can result in unilateral SSHL through either injury to the inner ear by Temporal bone fractures (mainly transverse fractures) or Penetrating injuries that damage the otic capsule or sublux the stapes into the vestibule, which can result in a perilymphatic fistula or Labyrinthine 20.

Neoplasm must be ruled out in unilateral hearing loss. Early diagnosis of lesions of the internal auditory meatus or cerebellopontine angle requires special attention. The importance of early diagnosis of these lesions is stressed with regard to the mortality rate for larger tumors and to the preservation of facial nerve function 3. Vestibular schwannoma, also known as acoustic neuroma, is the most common type of neoplasm to cause SNHL and accounts for $80 \%$ of CPA neoplasms 43 . Although vestibular schwannoma typically causes a progressive unilateral hearing loss, 10 to $26 \%$ of cases initially present as a sudden SNHL 44,45 . The remaining $20 \%$ of CP angle masses include meningioma, dermoid cysts, arachnoid cysts, hemangiomas, cholesterol granulomas and metastatic lesions.

The gold standard diagnostic modality in cerebellopontine angle tumor is magnetic resonance imaging (MRI) with gadolinium-enhancement and it help to detect evidence of central demyelination, intracochlear hemorrhage lesion or vascular. Auditory brain stem response (ABR) also can be helpful in identifying patients at high risk but can fail to detect tumors that are smaller than $1 \mathrm{~cm}$ in diameter $11,39,40,41$. As well as, there is no relationship between hearing loss and tumor size 5. Magnetic resonance imaging is recognized as the gold standard for the evaluation of these problems, but if a complete examination of the internal auditory canals and head is done on every patient, the cost is high. Although less expensive, screening with audiometric brain stem response risks missing up to $33 \%$ of small tumors 14 . The limitation of health care resources challenges otolaryngologists to develop reasonable cost-containment guidelines for the evaluation of patients with SNHL for the presence of retro cochlear pathology 9. On the other hand, auditory brainstem response (ABR) is a good screening test for cerebellopontine angle (CPA) tumors and other retro cochlear diseases. The pathologic correlates of many cases with positive ABR remain unknown 10.

To compare audiometric parameters in patients with vestibular schwannoma and in those with hearing loss from other causes; and to assess proposed screening criteria by comparing published protocols. As regards vestibular schwannoma screening protocols, the best compromise between sensitivity and screening rate 12 . Different pattern of hearing loss can occur with cerebellopontine angle (CPA) tumors, but it typically causes high-frequency SNHL with word recognition is significantly reduced and is typically not in agreement with the pure-tone thresholds 20. In our study, 7 cases retrocochlear pathology on MRI report, 5 cases of them have cerebellopontine angle (CPA) tumors: two cases were Vestibular schwannoma, one case was arachnoid cyst and remaining two cases was without clear radiological diagnosis. 2 of those partially absent wave-V and 3 of those have delay in latency of wave-V with interaural delay of wave-V more than $0.2 \mathrm{~ms}$. Jack D. et al, he finds the Falsenegative responses are few and false-positive responses are common. Brain stem electric response audiometry BERA is the most efficient audiometric test available today in our search for tumors affecting the auditory nerve (13).

\section{Conclusion}

After define a patient with USNHL (USNHL) or single-sided hearing loss need to identify the possible risk and cause of this hearing loss and to make early clear diagnosis and management plan. All patients with USNHL need full audiological screening. Regarding our study, we recommend MRI as part of their diagnostic workup to early role out cerebellopontine angle lesion. The choice of diagnostic laboratory tests should be directed by patient risk factors and exposures. Few studies focused on adult unilateral SNHL and most of researches are advised further studies required in this field.

\section{References}

1. Reiss M, Reiss G (2000) Differential diagnosis of unilateral hearing loss. Praxis 89(6): 241-247.

2. (2015) American Academy of Audiology Clinical Practice Guidelines Adult Patients with Severe-to- Profound Unilateral Sensorineural Hearing Loss.

3. Dokianakis G, Ferekidis E, Pantazopoulos P (1978) [Unilateral deafness (author's transl)]. Arch Otorhinolaryngol 219(2): 351-353.

4. Voelker C, Chole R (2010) Unilateral sensorineural hearing loss in adults: etiology and management. In Valente M, Oeding K (eds.) Fitting Options for Children and Adults with Single-Sided Deafness, Sem Hear 31(4): 313-325.

5. Saunders JE, Luxford WM, Devgan KK, Fetterman BL (1995) Sudden hearing loss in acoustic neuroma patients. Otolaryngol Head Neck Surg 113(1): 23-31.

6. Heman Ackah SE, Jabbour N, Huang TC (2010) Asymmetric sudden sensorineural hearing loss: is all this testing necessary? J Otolaryngol Head Neck Surg 39(5): 486-490.

7. Kuhn M, Heman Ackah SE, Shaikh JA, Roehm PC (2011) Sudden Sensorineural Hearing Loss: A Review of Diagnosis, Treatment, and Prognosis. Trends Amplif 15(3): 91-105.

8. Hendrix R, Dedio R, Selafani A (1990) The use of diagnostic testing in asymmetric sensorineural hearing loss. Otolaryngol Head Neck Surg 103(4): 593-598.

9. Urben SL, Benninger MS, Gibbens ND (1999) Asymmetric sensorineural hearing loss in a community based population. Otolaryngol Head Neck Surg 120(6): 809-814.

10. Bu-Saba N, Rebeiz E, Salman S, Thornton A, West C (1994) Significance of false-positive auditory brainstem response: A clinical study. Am J Otol 15(2): 233-236. 
11. Schick B, Brors D, Koch O, Schäfers M, Kahle G (2001) Magnetic resonance imaging in patients with sudden hearing loss, tinnitus and vertigo. Otol Neurotol 22(6): 808-812.

12. Gimsing S (2010) Vestibular schwannoma: when to look for it? J Laryngol Otol 124(3): 258-264.

13. Jack D Clemis, Therese (1979) Brain stem electric response audiometry in the differential Diagnosis of acoustic tumors. Laryngoscope 89(1): 31-42.

14. Carrier DA, Arriaga MA (1997) Cost-effective evaluation of asymmetric sensorineural hearing loss with focused magnetic resonance imaging. Otolaryngol Head Neck Surg 116(6): 567-574.

15. Arts HA (2010) Sensorineural hearing loss in adults. In: Flint PW, Haughey BH, Lund VJ, et al., (Eds.) Cummings Otolaryngology: Head and Neck Surgery ( $5^{\text {th }}$ edn.), Philadelphia, PA: Elsevier 2117-2127.

16. Divyank Bansal, Saurabh Varshney, Manu Malhotra, Poonam Joshi, Narendra Kumar (2016) Unilateral sensorineural hearing loss retrospective study. Indian j otol 22(4): 262-267.

17. Sharma M, Singh P, Kapoor M, Goel M (2015) Pattern of sensorineural hearing loss in patients attending ENT OPD. Int J Oral Health Med Res 2(1): 5-8.

18. Varshney S (2016) Unilateral sensorineural hearing loss(USNHL)-Still a challenge to manage. Otolaryngol Int 1: 19-22.

19. Hughes GB, Freedman MA, Haberkamp TJ, Guay ME (1996) Sudden sensorineural hearing loss. Otolaryngol Clin North Am 29: 393-405.

20. Arts HA. Sensorineural hearing loss: evaluation and management in adults. In: Cummings CW Cummings: Otolaryngology Head and Neck Surgery. $4^{\text {th }}$ edn. Philadelphia, PA; Mosby 2005: 3535-3555.

21. Sande MA, Mandell GL (1981) Antimicrobial agents. In: Gilman AG, Goodman LS, Gilman A. The Pharmacological Basis of Therapeutics. Macmillan, New York, USA 70(5): 581.

22. Hallberg OE (1956) Sudden deafness of obscure origin. Laryngoscope 66(10): 1237-1267

23. Berrocal JR, Ramírez-Camacho R (2002) Sudden sensorineural hearing loss: supporting the immunologic theory. Ann Otol Rhinol Laryngol 111(11): 989-997.

24. Schuknecht HF (2001) Pathology of the Ear ( $2^{\text {nd }}$ edn,). Philadelphia, PA; Lea and Febiger. Otol Neurotol 22: 113-122.

25. Hanner P, Rosenhall U, Edström S, Kaijser B (1989) Hearing impairment in patients with antibody production against Borrelia burgdorferi antigen. Lancet 1(8628): 13-15.

26. Dolan S, Everett ED, Renner L (1986) Hearing loss in Rocky Mountain spotted fever. Ann Intern Med 104(2): 285.

27. Steinfeld HJ, Silverstein J, Weisburger W, Rattner (1988) Deafness associated with Rocky Mountain spotted fever. Md Med J 37(4): 287288.

28. Grénman R (1985) Involvement of the audiovestibular system in multiple sclerosis. An otoneurologic and audiologic study. Acta Otolaryngol Suppl 420: 1-95.
29. Noffsinger D, Olsen WO, Carhart R, Hart CW, Sahgal V (1972) Auditory and vestibular aberrations in multiple sclerosis. Acta Otolaryngol Suppl 303: 1-63.

30. Drulović B, Ribarić-Jankes K, Kostić V, Sternić N (1994) Multiple sclerosis as the cause of sudden "pontine" deafness. Audiology 33(4): 195-201.

31. Franklin DJ, Coker NJ, Jenkins HA (1989) Sudden sensorineural hearing loss as a presentation of multiple sclerosis. Arch Otolaryngol Head Neck Surg 115(1): 41-45.

32. Daugherty WT, Lederman RJ, Nodar RH, Conomy JP (1983) Hearing loss in multiple sclerosis. Arch Neurol 40(1): 33-35.

33. Curé JK, Cromwell LD, Case JL, Johnson GD, Musiek FE (1990) Auditory dysfunction caused by multiple sclerosis: detection with MR imaging. AJNR Am J Neuroradiol 11(4): 817-820.

34. Armington WG, Harnsberger HR, Smoker WR, Osborn AG (1988) Normal and diseased acoustic pathway: evaluation with MR imaging. Radiology 167(2): 509-515.

35. Gebarski SS, Gabrielsen TO, Gilman S, Knake JE, Latack JT, et al. (1985) The initial diagnosis of multiple sclerosis: clinical impact of magnetic resonance imaging. Ann Neurol 17(5): 469-474.

36. JR Kearns (1977) Presbycusis. Can Fam Physician 23: 96-100.

37. Schuknecht HF, Kirchner JC (1974) Cochleaz MRI era. 1993; 103(4 Pt 1) 431-otosclerosis: factor 436.fantasy. Laryngoscope 84: 766-782.

38. Katherine M Smith (2014) Children At-Risk For Hearing Impairment: A Retrospective Study of The Ontario Infant Hearing Program Population.

39. Fitzgerald DC, Mark AS (1998) Sudden hearing loss: frequency of abnormal findings on contrast- enhanced MR studies. AJNR Am J Neuroradiol 19(8): 1433-1436.

40. Aarnisalo AA, Suoranta H, Ylikoski J (2004) Magnetic resonance imaging findings in the auditory PATHWAY of patients with sudden deafness. Otol Neurotol 25(3): 245-249.

41. Shinohara S, Yamamoto E, Saiwai S, Tsuji J, Muneta Y, et al. (2000) Clinical features of sudden hearing loss associated with a high signal in the labyrinth on unenhanced T1-weighted magnetic resonance imaging. Eur Arch Otorhinolaryngol 257(9): 480-484.

42. Saunders JE, Luxford WM, Devgan KK, Fetterman BL (1995) Sudden hearing loss in acoustic neuroma patients. Otolaryngol Head Neck Surg 113(1): 23-31.

43. Zulch KJ. Epidemiology of brain tumors: general statistical and biological data. In: Zulch KJ Brain Tumors: Their Biology and Pathology ( $3^{\text {rd }}$ edn.). Berlin, Germany; Springer-Verlag 1986: 85-112.

44. Moffat DA, Baguley DM, von Blumenthal H, Irving RM, Hardy DG (1994) Sudden deafness in vestibular schwannoma. J Laryngol Otol 108(2): 116-119.

45. Selesnick SH, Jackler RK, Pitts LW (1993) The changing clinical presentation of acoustic tumors in the MRI era. Laryngoscope 103(4): 431-436. 
This work is licensed under Creative Commons Attribution 4.0 License DOI: $10.19080 / G J O .2018 .15 .555919$

\section{Your next submission with Juniper Publishers} will reach you the below assets

- Quality Editorial service

- Swift Peer Review

- Reprints availability

- E-prints Service

- Manuscript Podcast for convenient understanding

- Global attainment for your research

- Manuscript accessibility in different formats

( Pdf, E-pub, Full Text, Audio)

- Unceasing customer service

Track the below URL for one-step submission https://juniperpublishers.com/online-submission.php 